\title{
Genesis of Non-Coding RNA Genes in Human Chromosome 22-A Sequence Connection with Protein Genes Separated by Evolutionary Time
}

\author{
Nicholas Delihas $\mathbb{1}$ \\ Department of Microbiology and Immunology, Renaissance School of Medicine, Stony Brook University, \\ Stony Brook, New York, NY 11794-5222, USA; Nicholas.delihas@stonybrook.edu \\ Received: 16 July 2020; Accepted: 1 September 2020; Published: 3 September 2020

\begin{abstract}
A small phylogenetically conserved sequence of 11,231 bp, termed FAM247, is repeated in human chromosome 22 by segmental duplications. This sequence forms part of diverse genes that span evolutionary time, the protein genes being the earliest as they are present in zebrafish and/or mice genomes, and the long noncoding RNA genes and pseudogenes the most recent as they appear to be present only in the human genome. We propose that the conserved sequence provides a nucleation site for new gene development at evolutionarily conserved chromosomal loci where the FAM247 sequences reside. The FAM247 sequence also carries information in its open reading frames that provides protein exon amino acid sequences; one exon plays an integral role in immune system regulation, specifically, the function of ubiquitin-specific protease (USP18) in the regulation of interferon. An analysis of this multifaceted sequence and the genesis of genes that contain it is presented.
\end{abstract}

Keywords: de novo gene birth; gene evolution; protogene; long noncoding RNA genes; pseudogenes; USP18; GGT5; Alu sequences

\section{Introduction}

The genesis of genes has been a major topic of interest for several decades [1,2]. One mechanism of gene formation is by duplication of existing genes [1,3]. This is considered one of the major processes in protein gene development, but it has also been shown that there is a prevalence of gene birth from noncoding DNA via de novo processes [4-7]; this pathway also significantly contributes to new protein gene formation [4,7]. Working with yeast Saccharomyces cerevisiae genomic segments, Carvunis et al. [4] formulated an evolutionary model for the de novo development of protein genes in genetic regions where there are no annotated genes but where there is the translation of small open reading frames. These regions are considered protogene elements that can develop into functional genes. With respect to long noncoding RNA (lncRNA) genes, Ulitsky and Bartel [8] have provided a comprehensive background on lncRNA transcripts and genes that includes a discussion of mechanisms of lncRNA gene origins where some gene birth processes may be similar to those that operate in protein gene formations.

In this treatise, we analyze the development of long intergenic noncoding RNA (lincRNA) genes and pseudogenes by an evolutionarily conserved ancestral sequence. This is a repeat element found in human chromosome 22. It was previously termed clincRNA [9] and is now officially termed FAM247 by the HUGO Gene Nomenclature Committee (https://www.genenames.org/), and it constitutes the FAM247A-D gene family. The FAM247A gene sequence has previously been used as a guide to finding homologous sequences [9]. Heretofore, FAM247 is used in place of FAM247A. 
We previously proposed that FAM247 carries information to form a nucleation site for gene development [9]. This is best exemplified with the formation of pseudogenes by the addition of extraneous chromosomal sequences to specific sites on FAM247, a process that is now described here in this current paper. This is a model that perhaps can be considered analogous to the model of de novo protein gene development via a protogene element [4], with the FAM247 sequence serving as the protogene. During the formation of these pseudogenes, sequences that consist of full or partial copies of unprocessed parent protein genes are added to the FAM247 protogene, as well as sequences that are added from other unrelated genomic regions to form the final gene sequence.

Both the pseudogenes and the FAM247A-D lincRNA family genes appear to be human-specific. The FAM247 sequence is also found in protein genes USP18 (ubiquitin-specific protease) and GGT5 (gamma glytamyltransferase). Both these genes date back in evolutionary time, USP18 over 350 million years ago (MYA) and GGT5 over 90 MYA. Thus, the FAM247 sequence has formed a part of genes through much of vertebrate evolution, and it continues to do so. The FAM247 sequence is of particular interest because of its evolutionary conservation and its presence in diverse genes, from humans to zebrafish. In addition, the FAM247 sequence carries information in terms of its open reading frames where the open reading frames are found to form exon sequences of proteins, the most important being the carboxy terminal amino acid sequence of USP18.

\section{Background on Conserved Linked Sequences}

FAM247 is present in different segmental duplications or low copy repeats (LCR22) in human chromosome 22 (chr22) as part of phylogenetically conserved linked gene sequences [9]. Figure 1 is a representation of these linked sequences, and it shows conserved nearest neighbor sequence signatures found in humans. The linked gene sequences are repeated in chr22 and generate gene families. The signatures are also representative of ancestral primate linked sequences, e.g., the sequence arrangement in Figure $1 \mathrm{~b}$ is present in the Rhesus Old World monkey (Macaca mulatta), where FAM247 and spacer sequences are linked to GGT1 on chr10. The spacer sequence (3953 bp) depicted in Figure 1 is also conserved in primates. It is present in Pan troglodytes (chimpanzee), Papio Anubis (olive baboon), Pongo abelii (Sumatran orangutan), and Macaca mulatta (Rhesus monkey) genomes, but it does not encode genes or form part of genes. This conservation indicates it may have a function. FAM247 is the common denominator in Figure 1a,b. In Figure 1c, the FAM247 sequence depicted is embedded in the USP18 gene.

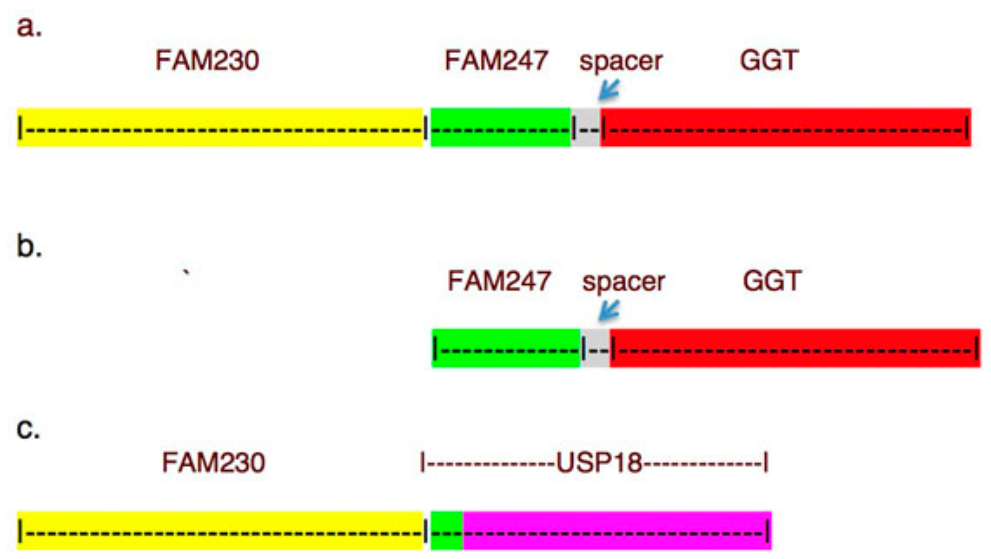

Figure 1. Schematic representation of evolutionarily conserved linked sequences with different colors depicting different sequences and family genes, as described in [9]. (a,b) Conserved sequences that are found linked to GGT sequences. (c) The FAM247 sequence (green highlight) is embedded in the USP18 gene. 
Table 1 contains a list of human gene families that are found in repeat units shown in Figure 1 and indicates the sequence or chromosomal locus of origin. For example, GGT represents the locus of origin of GGT1, the gamma-glutamyltransferase and gamma-glutamyltransferase light chain genes and their respective pseudogenes; FAM247 is the sequence/locus of origin of GGT5.

Table 1. Human genes and gene families found in linked sequences: FAM230-FAM247-GGT, FAM230-USP18.

\begin{tabular}{ccc}
\hline Gene/Gene Family & Type & Locus Origin \\
\hline${ }^{*}$ FAM230A-J & lincRNA & FAM230 \\
FAM247A-D & lincRNA & FAM247 \\
POM121L9P, POM121L10P & pseudogene & FAM247 \\
BCRP3 & pesudogene & FAM247 \\
GGT1, GGT2 & protein & GGT \\
GGTLC2 & protein & GGT \\
GGTLC3 & protein & GGT \\
GGT3P & pseudogene & GGT \\
GGT4P & pseudogene & GGT \\
GGTLC4P & pseudogene & GGT \\
GGTLC5P & pseudogene & GGT \\
GGT5 & protein & FAM247 \\
USP18 & protein & FAM247
\end{tabular}

* Some FAM230 family members, such as FAM230J, do not have the linked sequence signatures.

A description of genes is as follows. POM121L9P and POM121L10P are members of the POM121 transmembrane nucleoporin like 1 pseudogene family. BCRP3 is a member of the BCR pseudogene family. $B C R$, a large gene of $137,529 \mathrm{bp}$, is the activator of RhoGEF and GTPase and was formerly termed breakpoint cluster region protein. The $B C R$ gene is important clinically as it is associated with the production of the Philadelphia chromosome in chronic myelogenous leukemia [10,11]. POM121L9P and BCRP3 stem from the FAM247 sequence at chromosomal loci, where the GGT sequence is found, as represented in Figure 1b. USP18 is the ubiquitin-specific peptidase gene, a member of the deubiquitinating protease family; the protein product plays a major role in interferon regulation [12], and it has multiple functions [13].

\section{3. lincRNA Gene Families}

The FAM230 lincRNA and FAM247 lincRNA gene families were named by the HUGO Gene Nomenclature Committee (https://www.genenames.org/) [14]. These genes exemplify how segmental duplications or low copy repeats in chromosome 22 are a driving element in the genesis and proliferation of lincRNA gene families. Ten FAM230 and five FAM247 genes are present in chr22 low copy repeats (LCR22) that originate from sequence duplications [9]. FAM230 family genes differ from one another in sequence, transcript sequence and exon number, and RNA expression in various fetal developing tissues $[9,15,16]$. Their functions are not known. FAM230 sequences are also present in primates, but these sequences are annotated as predicted protein genes or pseudogenes, not as lincRNA genes, e.g., more than eleven genes that contain the FAM230 sequence in chimpanzee and gorilla are annotated as protein genes and two FAM230 sequences in Rhesus monkey and olive baboon are annotated as pseudogenes. An example is LOC106992440, which is found in the Rhesus monkey, annotated as an uncharacterized pseudogene, and resides in a chr locus that is homologous to that of human FAM230D linked to USP18 in humans; the Rhesus LOC106992440 has 58\% identity with the human lincRNA FAM230D. In this and other cases, the detection of an experimental transcript that verifies the computational prediction of a protein gene or pseudogene is essential. Evolutionarily, the FAM230 sequence may have originated in the Rhesus monkey or Old World monkeys as the FAM230 sequence is present in the Rhesus genome but is not found in the genome of the Prosimian primate ancestor, Philippine tarsier. 
The FAM247 lincRNA gene family may have newly formed in humans as there are few or no differences in gene sequence or RNA transcript expression in somatic and fetal developing tissues $[9,15]$. A homologous sequence to FAM247 is present in chimpanzee and is linked to GGT2. It has the full-length FAM247 sequence [9], but the chimpanzee sequence has not been annotated as a gene. Segments of sequences homologous to FAM247 are found in other primate genomes (gorilla, orangutan, Rhesus monkey, Philippine tarsier), and the FAM247 sequence may date back evolutionarily to the house mouse and zebrafish (discussed below).

\section{The FAM247 Sequence is Present in Diverse Genes}

A significant property of the FAM247 sequence is that it forms part of diverse genes. Sequences homologous to FAM247 form genes that include lincRNA genes, pseudogenes, and protein genes (Figure 2). These genes stem from phylogenetically conserved nearest neighbor gene loci, where the FAM247 sequence is linked to adjacent genes that form signatures containing gene families, e.g., FAM230E-FAM247C-GGT3P [9] present in segmental duplication LCR22A and FAM230B-FAM247A-GGT2 in LCR22D. Other than the FAM247 lincRNA family genes, which contain the entire $11,231 \mathrm{bp}$, only segments of FAM247 are found to be part of other genes. The ends of these segments may represent sequence breaks, i.e., bp positions $\sim 5958$ and $~ 8000-8200$ (Figure 2, see numbers above green highlighted FAM247). These are regions that contain Alu sequences (Figure 2, caption), and may provide sites for attachment of other sequences to FAM247. FAM247 contains a total of fifteen Alu elements.

\section{Diverse genes formed from FAM247 sequence}

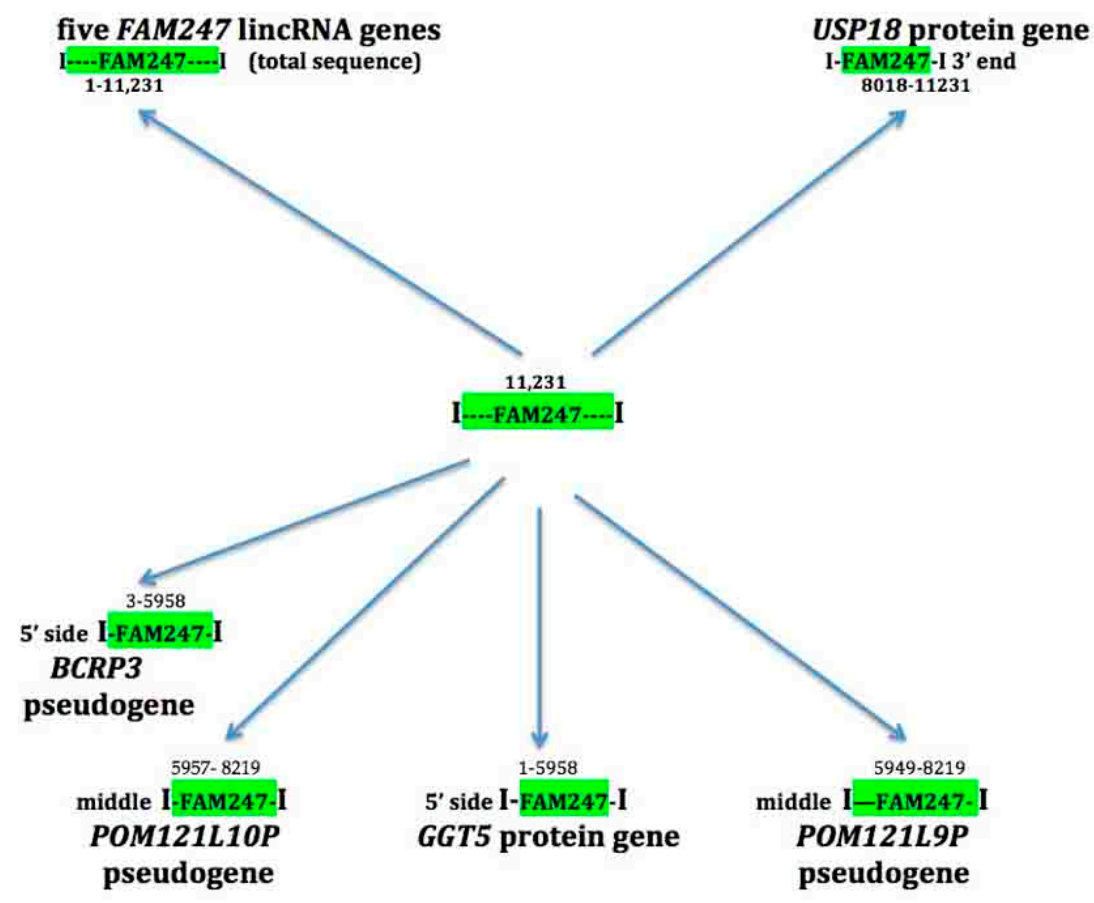

Figure 2. Protein genes, lincRNA genes, and pseudogenes that stem from the FAM247 sequence and contain different sections of the FAM247 sequence (shown in bp position numbers above FAM247 highlighted in green). Analysis of FAM247 by the RepeatMasker program: RepeatMasker http: //www.repeatmasker.org/cgi-bin/WEBRepeatMasker shows the presence of Alu sequences in the FAM247 sequence at bp positions 6007-6285 and 8063-8374, the regions close to breaks. The segment that includes bp positions 8063-8374 of FAM247 has seven Alu elements in tandem repeats. 


\subsection{USP18}

A comparison of USP18 chromosomal coordinates at loci in different species shows that the position is evolutionarily conserved relative to adjacent genes (Figure 3). This provides nearest neighbor signatures where evolutionary history (genetic synteny) and origins of USP18 can be assessed. Two neighbor genes, PEX26 (peroxisomal biogenesis factor 26) and TUBA8 (tubulin alpha 8) are in homologous loci that show a conserved orientation with respect to each other in the chromosomes of mice (Mus musculus (house mouse)) and primates (Figure 3a-c). In zebrafish (Danio rerio, a member the Cyprinidae family of freshwater fish), the tubulin gene (termed tuba814 tubulin, alpha 8 like 4) appears to have moved to a different chromosome and developed into two genes, TUBA $a$ and TUBAb; this results in PEX28 and USP18 as immediate neighbor genes in zebrafish chr4 (Figure 3d). The nearest neighbor history of the USP18 gene locus and the display of evolutionary conservation of gene positions relative to each other are consistent with a common lineage of the USP18 gene. Previously, Ulitsky and Bartel [8] provided an interesting analysis of human, mouse, and zebrafish vertebrate genomes with respect to the concept of synteny of genetic loci and the lineage of lincRNA genes through evolution.

a.

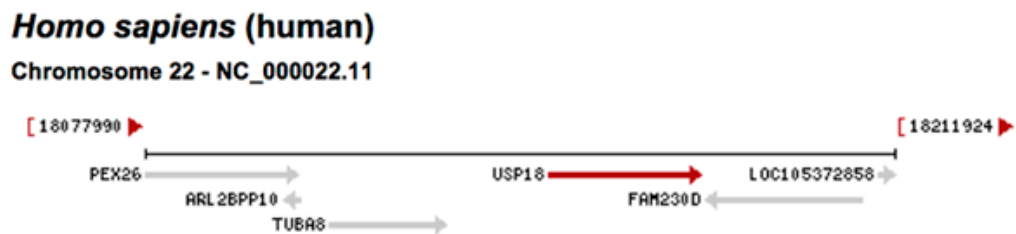

b.

Pan troglodytes (chimpanzee)

Chromosome 22 - NC_036901.1

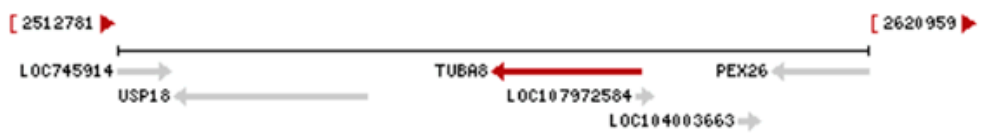

c.

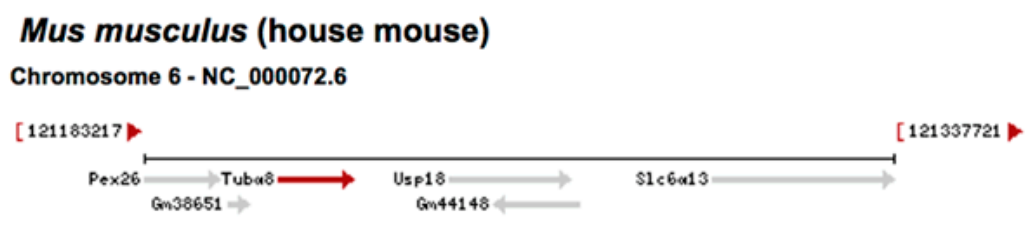

d.

Danio rerio (zebrafish)

Chromosome 4 - NC_007115.7

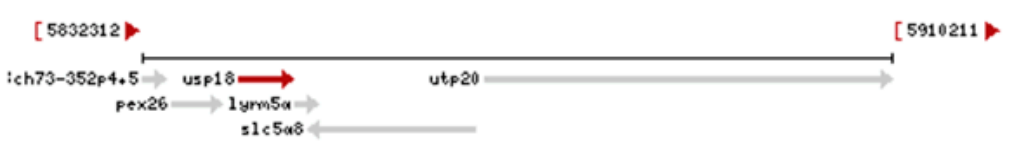

Figure 3. Genes that are adjacent to USP18 are found in different species and are shown in the chromosomal and gene maps. (a-d) Drawings of gene arrangements are taken directly from the NCBI website: https://www.ncbi.nlm.nih.gov/gene [17].

To analyze the phylogenetic relatedness of USP18 gene nt and aa sequences, sequences were aligned from zebrafish, the house mouse, three primate species, and humans. The resultant percent sequence identities mimic evolutionary distances between the species (Table 2), with a linear change in $\mathrm{nt}$ and aa sequences with time between the primate and mouse species (not shown). The pattern shows 
a continuum of gene nt and protein aa sequence change with evolutionary time and is consistent with a common lineage of the USP18 gene that dates to an ancestor of zebrafish, more than 350 million years ago (MYA). This parallels the nearest neighbor gene history of USP18.

Table 2. USP18 gene and protein sequence identities and evolutionary time between species.

\begin{tabular}{cccc}
\hline Species & $\begin{array}{c}\text { USP18 Gene nt } \\
\text { Sequence \%Identity }\end{array}$ & $\begin{array}{c}\text { USP18 aa Sequence } \\
\text { \%Identity }\end{array}$ & $\begin{array}{c}\text { Evolutionary Age } \\
\text { (MYA)* }\end{array}$ \\
\hline human & $100 \%$ & $100 \%$ & 0 MYA \\
chimpanzee & $99 \%$ & $99 \%$ & 6 MYA \\
Rhesus monkey & $92 \%$ & $94 \%$ & 25 MYA \\
Philippine tarsier & $66 \%$ & $80 \%$ & 50 MYA \\
mouse & $51 \%$ & $71 \%$ & 90 MYA \\
zebrafish & $39 \%$ & $31 \%$ & 350 MYA \\
\hline
\end{tabular}

* approximate age in million years ago (MYA) [18].

\subsection{USP18 Exon 11}

Both human exon 11, which encodes the last 14 aa (the carboxy terminal end) of the USP18 peptidase, and the $3^{\prime}$ UTR of the USP18 mRNA sequence are provided by the FAM247 sequence [8]. The identity between the FAM247 nt sequence and the human/primate exon11 nt sequences is $100 \%$, with the exception of that of Philippine tarsier (Figure 4). The sequence of the carboxy terminal exon is more stable than that of the sequence of the entire gene (compare with Table 2). The identities of the USP18 3'UTR sequences from various species, compared to FAM247 (Table 3), shows the 3'UTR sequence is also conserved in primates, but to a lesser extent than that of exon 11, and is more similar to the USP18 gene.

a.

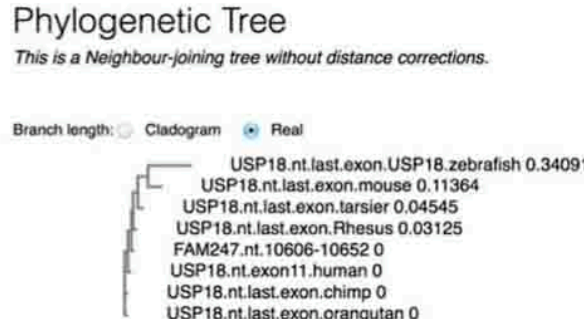

b.

Nucleotide sequence identities of last exon of USP18 compared to FAM247

\begin{tabular}{|l|l|}
\hline Source of nt sequence & $\begin{array}{l}\text { Percent identity } \\
\text { relative to FAM247 }\end{array}$ \\
\hline FAM247.nt.10606-10652 & 100 \\
\hline USP18.nt.exon11.human & 100 \\
\hline USP18.nt.last.exon.chimp & 100 \\
\hline USP18.nt.last.exon.orangutan & 100 \\
\hline USP18.nt.last.exon.Rhesus.monkey & 100 \\
\hline USP18.nt.last.exon.Philippine.tarsier & 91 \\
\hline USP18.nt.last.exon.mouse & 75 \\
\hline Usp18.nt.last .exon.zebrafish & 52.3 \\
\hline
\end{tabular}

c.

Alignment of nt sequences from last exon of USP18 and FAM247

USP18.nt.1ast.exon.USP18.zebrafiah USP18.nt.1ast.exon. mouse
USP18.nt.2ast. exon.tarsier FNM247.nt.10606-10652 USP18, nt. exon11, human USP18,nt, last, exon. orang USP18.nt.1ast.exon.Rhesus agaaacagectacttgettetgtacagaaagaagagtttgg 44 geaggaaactgcatacet tetggtttacatgaagattasgteet 44 ceaggaaactgcatatettotggtttacatgaagatggagtget 44 . geaggaaactgcatatettetggtttacatgaagatggagtgct 44 geaggaaactgegtatettetggtttacatgagatgagaatgct 44 
Figure 4. Alignment of the USP18 terminal exon nt sequences from seven species compared with the FAM247 sequence. Data were obtained using the EBI Clustal Omega sequence alignment and phylogeny programs. The EMBL-EBI Clustal Omega Multiple Sequence Alignment program [19] at website http://www.ebi.ac.uk/Tools/msa/clustalo/ was used for nt sequence alignment. (a) Phylogenetic tree of USP18 terminal exon sequences from seven species and the FAM247 sequence. (b) The percent identities created using Clustal 2.1. (c) Alignment of nt sequences. USP18 gene sequences were accessed from the NCBI website https://www.ncbi.nlm.nih.gov/gene [17].

Figure 5 shows the USP18 aa sequence percent identity, sequence alignments, and a phylogenetic tree produced from an alignment of USP18 terminal exon aa sequences from different species with the translated aa sequence of FAM247. Eight of the 14 amino acid residues that form the terminal exon are totally conserved from primates to zebrafish, together with the FAM247 translated aa sequence (Figure 5, bottom). The USP18 carboxy terminal peptide sequence interacts with the INFAR2 interferon receptor, and this sequence is an important regulator of IFN signaling [12]; in addition, the carboxyl end sequence functions in delSGlyation [13,20,21]. A mutation in L365 in the exon 11 sequence ${ }_{359}$ QETAYLL $_{365}$ VYMKMEC $_{372}$ abolishes deISGylation and INAFR2 binding [20,21]; $\mathrm{L}_{365}$ is one of the evolutionarily conserved amino acids of exon 11 (Figure 5). The mutation may alter the protein conformation that is necessary for USP18 to function. On the other hand, the high number of aa residues conserved, relative to the FAM247 translated aa sequence, further supports the proposal that the FAM247 sequence was present in zebrafish USP18.

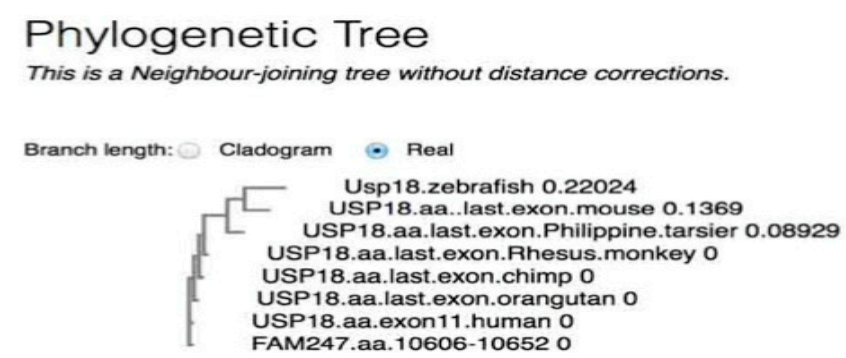

Amino acid sequence identities of last exon of USP18

\begin{tabular}{|l|l|}
\hline Source of aa sequence & $\begin{array}{l}\text { Percent identity } \\
\text { relative to FAM247 }\end{array}$ \\
\hline FAM247.aa.10606-10652 & 100 \\
\hline USP18.aa.exon11.human & 100 \\
\hline USP18.aa.last.exon.chimp & 100 \\
\hline USP18.aa.last.exon.orangutan & 100 \\
\hline USP18.aa.last.exon.Rhesus.monkey & 100 \\
\hline USP18.aa.last.exon.Philippine.tarsier & 78.6 \\
\hline USP18.aa..last.exon.mouse & 64.3 \\
\hline Usp18.zebrafish & 57.1 \\
\hline
\end{tabular}

Amino acid sequence of last exon of USP18

Figure 5. Alignment of the USP18 terminal exon amino acid sequences from seven species compared with the FAM247 translated amino acid sequence. Data were obtained using the EBI Clustal Omega sequence alignment and phylogeny programs. The EMBL-EBI Clustal Omega Multiple Sequence Alignment program [19] at website http://www.ebi.ac.uk/Tools/msa/clustalo/ was used for aa sequence alignment. (Top) Phylogenetic tree of USP18 terminal exon aa sequences from seven species and the FAM247 aa sequence. (Middle) The percent identities were created using Clustal 2.1. (Bottom) Alignment of aa sequences. 
The nt sequence similarity of 52\% between FAM247 and zebrafish last exon (Figure $4 \mathrm{~b}$ ), the presence of a number of invariant nt positions (Figure 4c), and the similarity with the $3^{\prime}$ UTR sequence (53\%; Table 3) suggests that this part of the FAM247 sequence was present in the USP18 sequence of zebrafish. The invariant nt residues of exon 11, e.g., positions nt 5-9 (Figure 4c), may relate to the functional importance of the USP18 carboxy terminal end in its role in the regulation of the immune system by USP18 [12,13]. These invariant nt positions may give a picture, albeit a small picture, of what the ancient FAM247 looked like.

Table 3. Nucleotide sequence identities of 3'UTR of USP18 from different species.

\begin{tabular}{cc}
\hline Source of nt Sequence & \% Identity Relative to FAM247 3' End \\
\hline FAM247A 3' end nt 10,653-11,231 & 100 \\
USP18 3'UTR human & 99.8 \\
USP18 3'UTR chimp & 98.6 \\
USP18 3'UTR Rhesus monkey & 90.2 \\
USP18 3'UTR Philippine tarsier & 71.9 \\
USP18 3'UTR mouse & 49.5 \\
USP18 3'UTR zebrafish & 53.1 \\
\hline
\end{tabular}

\subsection{GGT5}

The human GGT5 protein gene resides in chromosomal segmental duplication LCR22G and is linked to pseudogene POM121L9P with a spacer sequence, and the pseudogene GGTLC4P situated between GGT5 and POM121L9P (Figure 6) [9]. The GGT5 nearest gene/sequence arrangement is more complex than that of the signatures shown in Figure 1b. GGT5 is an anomaly as its sequence does not stem from a GGT locus, as other GGT family members do, but from the chromosomal site containing the FAM247 sequence [9]. GGT5 carries a sequence homologous to the $5^{\prime}$ half of the FAM247A sequence, bp positions 1-5958 (Figures 2 and 6), and POM121L9P contains part of the 3' half of FAM247 (bp 5949-8219). The FAM247 fragments are where there are or were Alu sequences. The GGTLC4P pseudogene derives its sequence from GGT (Figure 6).

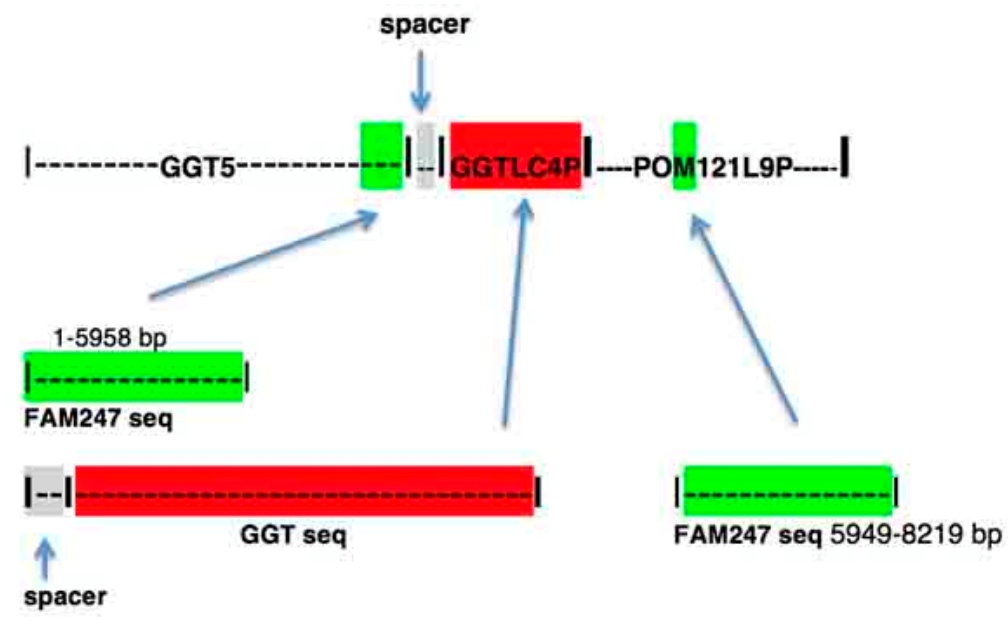

Figure 6. The genes linked to GGT5 in LCR22G with nearest neighbor arrangements (top schematic) and the source of sequences found in human-linked genes GGT5-GGTLC4P-POM121L9P.

GGT5 and POM121L9P appear to have been formed at very different evolutionary times. FAM247 is part of the GGT5 genes that are in nonhuman primates, including Philippine tarsier. In addition, FAM247 provides the sequence found in exon 1 of GGT5. There is a significant similarity between the FAM247 nt sequence and that of the mouse GGT5 exon 1 (Figure 7, top). There is not enough evidence to suggest that the mouse GGT5 contains the entire $5^{\prime}$ half of the FAM247 sequence, but the alignment of 
the mouse exon $1 \mathrm{nt}$ sequence with FAM247 shows that a significant number of nucleotides are invariant (Figure 7, bottom). Although there is invariance in 50 out of 173 nt between the FAM247 sequence and zebrafish GGT5 exon 1, the zebrafish exon sequence shows significant differences, which makes it difficult to further assess a sequence similarity. The exon 1 data are consistent with the formation of the GGT5 gene with the FAM247 sequence that occurred before the evolutionary appearance of primates and appearing in mice.

Percent identity of nucleotide sequences from GGT5 exon 1 compared to FAM247

\begin{tabular}{|l|l|}
\hline Source of exon 1 nt sequence & $\begin{array}{l}\text { Percent identity } \\
\text { relative to FAM247 }\end{array}$ \\
\hline FAM247 nt. 392-567 & 100 \\
\hline Exon 1 GGT5 human & 88 \\
\hline Exon 1 GGT5 Philippine tarsier & 80 \\
\hline Exon 1 GGT5 mouse & 69 \\
\hline Exon 1 GGT5b zebrafish & 46 \\
\hline
\end{tabular}

Alignment of nucleotide sequences from GGT5 exon 1 and FAM247

\begin{tabular}{|c|c|c|}
\hline exonl.cGr5b.zebratiah & -teteagtegaggegatgetgettttgtttaotegetttaqt & so \\
\hline exont.CGTs, mouse & atgget:qgggteacagggecaeqgtetgeetggtectge--.---tgggtgtaggteta & 54 \\
\hline 7ам247.392-567 & atggcecaggactacqgagecatggqtgacetggtect get gqggetggggetggggetg & 60 \\
\hline exonl.Philipplne, taraler & Atggeetqgqgetgeagggecateateageetggtectgetgqggeteqggetgggaetg & 60 \\
\hline \multirow[t]{2}{*}{ exonl. Ger5, human } & at $g 9$ cecqqggetacggggecaeggteagectagtectge -.....-tgggtetqgqgetg & 54 \\
\hline & ..... & \\
\hline exon1.GGT5b.zebratiah & gtgcactgetgcaattatetgeattegcatactt_...........-tteagcaancagaa & 98 \\
\hline exon1.cots, mouse & 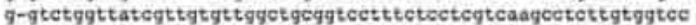 & 113 \\
\hline FAM247.392-567 & g-cgetggetgteattgtgetggetgtggtcetetetegacabeaggococatttgacec & 119 \\
\hline exoni.ptilippine,taraier & g-cactagtcateattgtgetggetgtggtectecetegecatcaggececetgtggece & 119 \\
\hline \multirow[t]{2}{*}{ exon1.GGrs, human } & g-egetgugetgteattgtgetggotgtgytcetetetegacaces ggeeceat qutggece & 113 \\
\hline & 0. & \\
\hline exonl.GGr5b.zebrafiah & atgegact ttactogggeeqetgtttetqcqgaeteteteatqugetcogacateqgeaq & 158 \\
\hline exont. cars, touse & eggtgocttcacgogtgetgeggtagcggetgactecaagatetgoteggatattggacg & 173 \\
\hline FN1247.392-567 & - coggectttgeceacgecgetgttgetgetgaetecangqutettoteabatattgtaeg & 178 \\
\hline exonl. Philippine.taraier & ecaggectttqgeceacgetgecattgctgctgactetaaggtetgeteggacattggace & 179 \\
\hline \multirow[t]{2}{*}{ exon1.cots. hrman } & ecaggectttgeecacgetgetgttgeegcegaetecaagqtetgeteggatattggacg & 173 \\
\hline & $\ldots \ldots, \cdots \cdots \cdots$ & \\
\hline exonl.GGrsb.zebrafi & 159 & \\
\hline exonl. cors tmotiae & 173 & \\
\hline FAM:247.392-567 & 178 & \\
\hline exonl.philippine.taraier & 180 & \\
\hline exonl. gets, human & 173 & \\
\hline
\end{tabular}

Figure 7. (Top) The percent identity of the FAM247 sequence with that of GGT5 exon 1 sequences from four species. Data were obtained using Clustal 2.1. (Bottom) Alignment of the GGT5 exon nucleotide sequences from the four species, compared with the FAM247 sequence, positions 192-567. Data were obtained using the EBI Clustal Omega sequence alignment and phylogeny programs. The EMBL-EBI Clustal Omega Multiple Sequence Alignment program [19] is at website http://www.ebi.ac.uk/Tools/ $\mathrm{msa} /$ clustalo/.

\subsection{Pseudogene POM121L9P}

POM121L9P has a very different sequence compared to the other POM121LP family pseudogenes, and it is a unique sequence. A schematic of the compositional make-up of the POM121L9P gene shows that it contains most of the sequence homologous to the putative parent gene, protein gene POM121L1 (LOC101929738 putative POM121-like protein 1, $2379 \mathrm{bp}$ ) on its $5^{\prime}$ side, and the BCRP1 pseudogene sequence (that is homologous to the $3^{\prime}$ section of the $B C R$ gene that includes $B C R$ terminal exons 19-23) on its 3' side (Figure 8). FAM247 may have formed a nucleation site for the addition of these motifs, which are copies of sequences from different regions of the genome, and developed the POM121L9P gene. The sequence motifs are found attached to $5^{\prime}$ and $3^{\prime}$ ends of FAM247 at FAM247 bp positions where there are Alu sequences (FAM247 bp positions 5949 and 8219). Additionally, the complete POM121L-1 sequence has an Alu sequence at bp positions 2309-2379 (the end of POM121L-1 is position 2304, at the attachment site with FAM247). Alu sequences may have facilitated the addition of POM121L-1 to FAM247. The BCR sequence addition to POM121L9P is more complex as there is an undefined sequence between the two (bp positions 4479-5779 on POM121L9P), and there are no Alu 
sequences detected in the BCR sequence at the junction site. The human POM121L9P pseudogene RNA transcript is highly expressed in somatic testis tissue, and there is a broad expression of circular RNAs in developing fetal tissues with major expression in lung and adrenal tissues $[15,16]$. Its functions are not known, but they should be of interest in view of the strong RNA expression levels.

\section{POM121L9P}

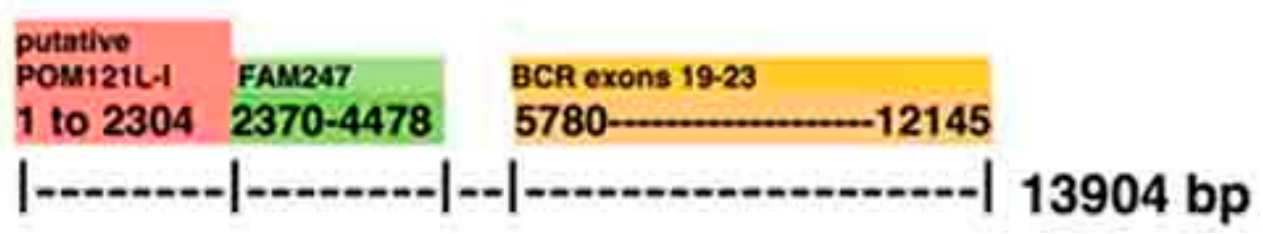

Figure 8. A schematic of the compositional make-up of the pseudogene POM121L9P. The numbers under the motifs shown represent the bp positions on the POM121L9P sequence. The FAM247 sequence that forms part of POM121L9P consists of FAM247 positions 5949-8219, where there are Alu sequences at both ends.

In a homologous nearest neighbor gene arrangement that is present in chimpanzee chr22, the genes are annotated as glutathione hydrolase light chain 2 gene (LOC749018) and putative POM121-like protein 1 gene (LOC112206778); these are linked to GGT5 through the spacer sequence (Figure 9). Thus, the human pseudogenes GGTLC4P and POM121L9P sequences are annotated as protein genes in the homologous chromosomal loci of chimpanzee. This is another example of human ncRNA gene sequences that are annotated as protein genes in nonhuman primates, but the isolation of protein products from the chimpanzee genes is essential to add any significance to it. Of importance is that $69 \%$ of the POM121L9P sequence is present in the chimpanzee genome with $98 \%$ identity at the genomic region where there is evolutionary synteny with the comparable chromosomal locus that resides in chimpanzee. There are no FAM247 or POM121L9P sequences that have been found linked to GGT5 in Rhesus. It appears that the development of the POM121L9P sequence may have begun in the chimpanzee but with a partial sequence.

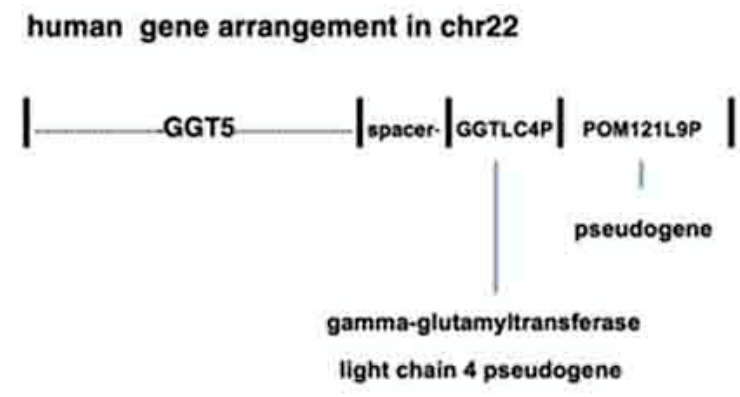

chimpanzee gene arrangement in chr22

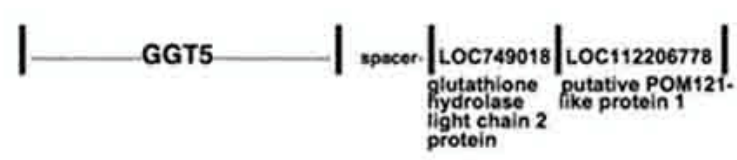

Figure 9. Nearest neighbor gene arrangements in human and chimpanzee chromosomal loci where the GGT5 gene resides.

\subsection{Pseudogenes BCRP3 and POM121L10P}

Human pseudogenes BCRP3 and POM121L10P are linked to GGT1 in the gene/sequence arrangement GGT1-spacer-BCRP3-POM121L10P, which is present in chr22 LCR22H. FAM247 forms 
part of the two pseudogenes: BCRP3, which has the FAM247 positions 33-5958 and POM121L10, positions 5957-8219 (Figure 2). Thus, parts of the $5^{\prime}$ and $3^{\prime}$ regions of FAM247 are found in these linked genes, which is similar to the presence of FAM247 in genes GGT5 and POM121L9P.

$B C R P 3$ is a member of the $B C R P$ pseudogene family consisting of eight pseudogenes, all of which contain the homologous sequence of the $3^{\prime}$ end sequence of the $B C R$ protein gene except for $B C R P 8$. $B C R P 3$ is one of the family members that differs as it contains additional sequence motifs (Figure 10) and is the only $B C R P$ family member that contains the FAM247 sequence. The BCRP3 gene appears to have a unique sequence. The compositional make-up of $B C R P 3$ shows that its $5^{\prime}$ side has the FAM247 sequence, which is followed by a 4255-bp segment of the immunoglobulin lambda locus (IGL) and the $3^{\prime}$ end of the BCR sequence (Figure 10). The IGL sequence (nt positions 590,381-594,292) from the Homo sapiens immunoglobulin lambda locus (IGL) on chromosome 22) [17] is homologous to the IGL locus V segments and three $\mathrm{C}$ segments, which are not known to encode immunoglobulin proteins. The IGL sequence has an Alu sequence at the junction with FAM247, which may relate to the process of attachment of IGL to FAM247 during the maturation of the pseudogene. The IGL sequence is made up entirely of $8 \mathrm{Alu}$ sequences, 7 LINE elements, other transposable elements, and small repeats (http://www.repeatmasker.org/cgi-bin/WEBRepeatMasker). In terms of RNA expression, the pseudogene shows a broad expression of linear RNA in 27 normal somatic tissues and a broad expression of circular RNA in developing fetal tissues $[15,16]$.

\section{BCRP3 Gene}

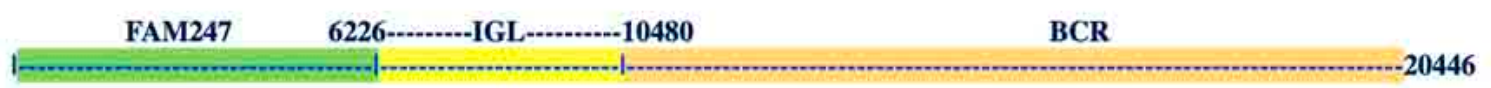

Figure 10. Sequence motif of the $B C R P 3$ gene. Positions 6226-10480 of $B C R P 3$ span the IGL insert. The total length of $B C R P 3$ is $20446 \mathrm{bp}$.

The POM121L10P sequence is linked to BCRP3 on chr22. It also contains the FAM247 sequence (Figure 2). POM121L10P is compositionally made up of nearly the entire sequence of the related pseudogene POM121L1P but has a 1062-bp sequence at its $3^{\prime}$ end that consists of a copy of the $3^{\prime}$ end of the BCR gene. POM121L10P also appears to be a unique gene construct. The POM121L10P linear RNA transcript is strongly expressed in testes; circular RNAs are broadly expressed in fetal tissues. $[15,16]$. Thus, both this gene and $B C R P 3$ show a robust RNA expression. It should be pointed out that there are additional POM121LP pseudogene family members that carry the FAM247 sequence, but they are not addressed here.

In the Rhesus genome, some genes/neighbor sequences display synteny. The Rhesus GGT1 is linked to the spacer sequence and followed by the FAM247 sequence, which is similar to that of the human GGT1 gene/sequence arrangement. Rhesus gene LOC107000612, annotated as a "breakpoint cluster region protein-like" is situated close to GGT1. This is part of the homologous chromosomal region where the pseudogene $B C R P 3$ resides in the human genome; approximately $78 \%$ of the human $B C R P 3$ sequence is present in the Rhesus genome at this locus. The BCRP3 sequence has not been detected in the early primate Philippine tarsier. Thus, the earliest appearance of the BCPR3 sequence is in the Rhesus species, and the sequence appeared to have matured into a pseudogene in humans. There was a large chromosomal expansion of the Rhesus monkey genome between genes GGT1 and GGT5. The chromosomal length between genes GGT1 and GGT5 in Philippine tarsier is $2872 \mathrm{bp}$; in the rhesus monkey, it is 216,200 bp. Thus, there is a 75-fold sequence expansion between GGT1 and GGT5 in Rhesus. Segments of the BCRP3 gene may have formed with this chromosomal expansion. This may account for the source of the $B C R P 3$ sequence in Rhesus, but again, the sequence is not found in Philippine tarsier.

Using Figures 8 and 10, a model of de novo gene development from protogene sequences can be visualized whereby the FAM247 sequence, which is present in genomic regions that display synteny, 
forms nucleation sites where other genomic sequences are added during the maturation process to complete the pseudogene structure.

\section{Conclusions}

Both the FAM247 lincRNA gene family and pseudogenes appear to have the FAM247 sequence as a foundation for gene development; however, the mechanism of formation and the compositional make-up between lincRNA genes and pseudogenes greatly differ. The FAM247 family (as well as the FAM230 lincRNA gene family) was formed by gene duplication and family members display sequences that are "variations on a theme". Although pseudogenes BCRP3, POM121L9P, and POM121L10P contain duplications of part of or entire portions of parent protein genes, they were formed differently by a de novo process of addition of large unrelated genomic sequences to the FAM247 sequence, with the resultant formation of unique pseudogene sequences. Alu elements are present in FAM247 at sites of attachment, and these may contribute to the process of sequence addition, possibly by $A l u / A l u$ recombination. As these pseudogenes are unique, with large sequences unrelated to the parent protein genes, the question is whether they should be called pseudogenes. How USP18 and GGT5 protein genes developed is not known, but a putative ancient FAM247 sequence was likely involved. A separate but important aspect of the FAM247 sequence in cellular and molecular functions is that it contributes the amino acid sequence for protein exons, the first exon of GGT5 and the last exon of USP18. The functions of the carboxy terminal aa sequence of USP18 are of major significance because of the important role in the regulation of the immune system.

A search for possible nucleation elements similar to FAM247 is important in order to determine the prevalence of this type of protogene. An analysis of repeat sequences that are part of phylogenetically conserved nearest-neighbor genes/sequences in human chromosomes that have a large number of segmental duplications, e.g., chr 15, 16, and X [22], may help find other gene forming elements. Blat searches with the Ensembl program using lncRNA gene sequences as a query can help locate sequences shared with other genes, including protein genes. Use of the NCBI Blast/align two sequences program may reveal small sequence segments that are present in diverse genes.

Funding: This research received no external funding.

Conflicts of Interest: The author declares no conflict of interest.

\section{References}

1. Ohno, S. Gene duplication and the uniqueness of vertebrate genomes circa 1970-1999. Semin. Cell Dev. Biol. 1999, 10, 517-522. [CrossRef]

2. Jacob, F. Evolution and tinkering. Science 1977, 196, 1161-1166. [CrossRef] [PubMed]

3. Wang, W.; Yu, H.; Long, M. Duplication-degeneration as a mechanism of gene fission and the origin of new genes in Drosophila species. Nat. Genet. 2004, 36, 523-527. [CrossRef]

4. Carvunis, A.R.; Rolland, T.; Wapinski, I.; Calderwood, M.A.; Yildirim, M.A.; Simonis, N.; Charloteaux, B.; Hidalgo, C.A.; Barbette, J.; Santhanam, B.; et al. Proto-genes and de novo gene birth. Nature 2012, 487, 370-374. [CrossRef] [PubMed]

5. McLysaght, A.; Guerzoni, D. New genes from non-coding sequence: The role of de novo protein-coding genes in eukaryotic evolutionary innovation. Philos. Trans. R. Soc. B Biol. Sci. 2015, 370, 20140332. [CrossRef]

6. Schlotterer, C. Genes from scratch-The evolutionary fate of de novo genes. Trends Genet. 2015, 31, 215-219. [CrossRef] [PubMed]

7. Van Oss, S.B.; Carvunis, A.R. De novo gene birth. PLoS Genet. 2019, 15, e1008160. [CrossRef] [PubMed]

8. Ulitsky, I.; Bartel, D.P. lincRNAs: Genomics, evolution, and mechanisms. Cell 2013, 154, 26-46. [CrossRef]

9. Delihas, N. Formation of human long intergenic non-coding RNA genes, pseudogenes, and protein genes: Ancestral sequences are key players. PLoS ONE 2020, 15, e0230236. [CrossRef]

10. Nowell, P.; Hungerford, D. A minute chromosome in human chronic granulocytic leukemia. Science 1960, 132, 1497. 
11. De Klein, A.; van Kessel, A.G.; Grosveld, G.; Bartram, C.R.; Hagemeijer, A.; Bootsma, D.; Spurr, N.K.; Heisterkamp, N.; Groffen, J.; Stephenson, J.R. A cellular oncogene is translocated to the Philadelphia chromosome in chronic myelocytic leukaemia. Nature 1982, 300, 765-767. [CrossRef] [PubMed]

12. Arimoto, K.I.; Löchte, S.; Stoner, S.A.; Burkart, C.; Zhang, Y.; Miyauchi, S.; Wilmes, S.; Fan, J.B.; Heinisch, J.J.; $\mathrm{Li}, \mathrm{Z}$; ; et al. STAT2 is an essential adaptor in USP18-mediated suppression of type I interferon signaling. Nat. Struct. Mol. Biol. 2017, 24, 279-289. [CrossRef] [PubMed]

13. Honke, N.; Shaabani, N.; Zhang, D.E.; Hardt, C.; Lang, K.S. Multiple functions of USP18. Cell Death Dis. 2016, 7, e2444. [CrossRef] [PubMed]

14. Bruford, E.A.; Braschi, B.; Denny, P.; Jones, T.E.M.; Seal, R.L.; Tweedie, S. Guidelines for human gene nomenclature. Nat. Genet. 2020, 52, 754-758. [CrossRef] [PubMed]

15. Szabo, L.; Morey, R.; Palpant, N.J.; Wang, P.L.; Afari, N.; Jiang, C.; Parast, M.M.; Murry, C.; Laurent, L.C.; Salzman, J. Statistically based splicing detection reveals neural enrichment and tissue-specific induction of circular RNA during human fetal development. Genome Biol. 2015, 16, 126. [CrossRef]

16. Fagerberg, L.; Hallström, B.M.; Oksvold, P.; Kampf, C.; Djureinovic, D.; Odeberg, J.; Habuka, M.; Tahmasebpoor, S.; Danielsson, A.; Edlund, K.; et al. Analysis of the human tissue-specific expression by genome-wide integration of transcriptomics and antibody based proteomics. Mol. Cell. Proteom. 2014, 13, 397-406. [CrossRef]

17. O’Leary, N.A.; Wright, M.W.; Brister, J.R.; Ciufo, S.; Haddad, D.; McVeigh, R.; Rajput, B.; Robbertse, B.; Smith-White, B.; Ako-Adjei, D.; et al. Reference sequence (RefSeq) database at NCBI: Current status, taxonomic expansion, and functional annotation. Nucleic Acids Res. 2016, 44, D733-D745. [CrossRef]

18. Siepel, A. Phylogenomics of primates and their ancestral populations. Genome Res. 2009, 19, $1929-1941$. [CrossRef]

19. Madeira, F.; Park, Y.M.; Lee, J.; Buso, N.; Gur, T.; Madhusoodanan, N.; Basutkar, P.; Tivey, A.R.N.; Potter, S.C.; Finn, R.D.; et al. The EMBL-EBI Search and Sequence Analysis Tools APIs in 2019. Nucleic Acids Res. 2019, 47, W636-W641. [CrossRef]

20. Malakhov, M.P.; Malakhova, O.A.; Kim, K.I.; Ritchie, K.J.; Zhang, D.E. Protein ISGylation Modulates the JAK-STAT Signaling Pathway. J. Biol. Chem. 2002, 277, 9976-9981. [CrossRef]

21. Dauphinee, S.M.; Richer, E.; Eva, M.M.; McIntosh, F.; Paquet, M.; Dangoor, D.; Burkart, C.; Zhang, D.E.; Gruenheid, S.; Gros, P. Contribution of increased ISG15, ISGylation and deregulated type I IFN signaling in Usp18 mutant mice during the course of bacterial infections. Genes Immun. 2014, 15, 282-292. [CrossRef] [PubMed]

22. Redaelli, S.; Maitz, S.; Crosti, F.; Sala, E.; Villa, N.; Spaccini, L.; Selicorni, A.; Rigoldi, M.; Conconi, D.; Dalprà, L.; et al. Refining the phenotype of recurrent rearrangements of chromosome 16. Int. J. Mol. Sci. 2019, 20, 1095. [CrossRef] [PubMed]

(C) 2020 by the author. Licensee MDPI, Basel, Switzerland. This article is an open access article distributed under the terms and conditions of the Creative Commons Attribution (CC BY) license (http://creativecommons.org/licenses/by/4.0/). 